Journal of Clinical and Nursing Research

Research Article

\title{
Photodynamic Therapy Combined with Electrosurgical Resection for Recurrent Bladder Cancer
}

\author{
Ziwei $\mathrm{Xu}^{1}$, Minhong $\mathrm{Wu}^{2}$, Lule $\mathrm{Wu}^{2}$, Xiaoxiong $\mathrm{Hu}^{2}$, Jianwen Sheng ${ }^{2}$, Yuwen $\mathrm{Wu}^{2}$, Huizhen $\mathrm{Fan}^{2 *}$ \\ ${ }^{1}$ Yichun University, Yichun 336000, Jiangxi Province, China; \\ ${ }^{2}$ Yichun People's Hospital, Yichun 336000, Jiangxi Province, China
}

\begin{abstract}
Bladder tumor is characterized by recurrent recurrence and distant metastasis, which determines the difficulty of completely curing bladder tumor. In recent years, the number of patients with bladder cancer is increasing, and the treatment of bladder cancer has become an important direction of clinical research.It is difficult to control bladder tumor by traditional therapy. Photodynamic therapy (PDT), as a new optical therapy, has gradually become the main method in clinical treatment of bladder tumor combined with transurethral resection of bladder tumor. In this paper, a patient with superficial recurrent bladder tumor was treated by photodynamic therapy combined with transurethral resection of bladder tumor. The advantages of photodynamic therapy in the treatment of bladder tumor and the selection of photosensitizer in the process of photodynamic therapy were discussed. After two recurrences, the patients chose photodynamic therapy. The tumors were resected one by one, and the wound was coagulated by roller electrode. After the drug was retained for 20 minutes, the bladder was empty. The spherical optical fiber was implanted into the bladder. The photodynamic energy was adjusted (light power $1.8 \mathrm{~W}$, light time 1302 s).There was no recurrence after operation.Most bladder tumors are superficial tumors, and bladder is a cavity organ, which determines that bladder is an ideal organ for photodynamic therapy. As a targeted drug, photosensitizer is only absorbed by bladder tumor after being perfused into bladder. The photosensitizer forms reactive oxygen species through oxygen and kills tumor cells. Clinical practice has proved that PDT has its unique advantages for superficial and recurrent bladder tumors. As the first generation photosensitizer, xipofen also has selectivity in the treatment of bladder cancer.
\end{abstract}

Key words: Bladder tumor; Photodynamic force; Transurethral resection of bladder tumor; Photosensitizer; Hiporfin

Publication date: September, 2020

Publication online:30 September, 2020

*Corresponding author: Huizhen Fan, fanfanyc@163. com

Bladder tumor is one of the most common malignant tumors in urology. In recent years, the number of bladder cancer patients in China has been increasing. Tumor recurrence and distant metastasis directly threaten the life and health of patients. The treatment of bladder cancer has become an important direction of clinical research $^{[1]}$. When it is difficult to control bladder tumor by traditional therapy, photodynamic therapy (PDT) is particularly effective in the treatment of bladder tumor ${ }^{[2]}$. In this paper, a patient with recurrent bladder tumor was successfully treated by photodynamic therapy combined with transurethral resection of bladder tumor. The advantages of photodynamic therapy in the treatment of bladder tumor and the choice of photosensitizer phoposide in the treatment were discussed.

\section{Summary of medical records}

Basic information of patients: A 90 year old woman was admitted to the hospital for another day after bladder cancer surgery for more than 10 years. The patient had no obvious inducement to have hematuria by naked eye more than 10 years ago, and showed intermittent whole course hematuria without blood clot. The diagnosis was 
bladder tumor. In 2006, the patient underwent partial cystectomy for bladder tumor;In 2013 and 2015, the patients underwent transurethral resection of bladder tumorNon invasive carcinoma of the urinary tract (low grade); In April 2019, because of the recurrence of bladder tumor, transurethral resection of bladder tumor was performed Noninvasive breast urothelial carcinoma (high grade); In July 2019, because of the recurrence of bladder tumor, the bladder resection was performed again, and the drugs were injected regularly after the operation. On December 10, 2019, the bladder tumor was resected with photodynamic therapy under general anesthesia. The operation process was as follows: The patients took the lithotomy position and disinfected the routine surgical site. The position and shape of bilateral ureteral openings were normal. Multiple new organisms were found in the right wall of bladder and anterior wall of bladder, with the size of $0.3 \mathrm{~cm}-0.5 \mathrm{~cm}$. The tumor was resected one by one, reaching to the muscular layer. The wound was coagulated by roller electrode. Because the tumor was small, it was not sent to pathology, and there was no obvious residual tissue. After the drug was reserved for 20 minutes, the bladder was empty. The spherical optical fiber was implanted into the bladder, and the photodynamic energy was adjusted (light power $1.8 \mathrm{~W}$, There was no bleeding in the bladder after the end of light irradiation. Anesthesia was satisfactory during the operation, the patient was in good condition, the vital signs were stable, the operation was smooth, and he returned to the ward safely(Figure 1-2).

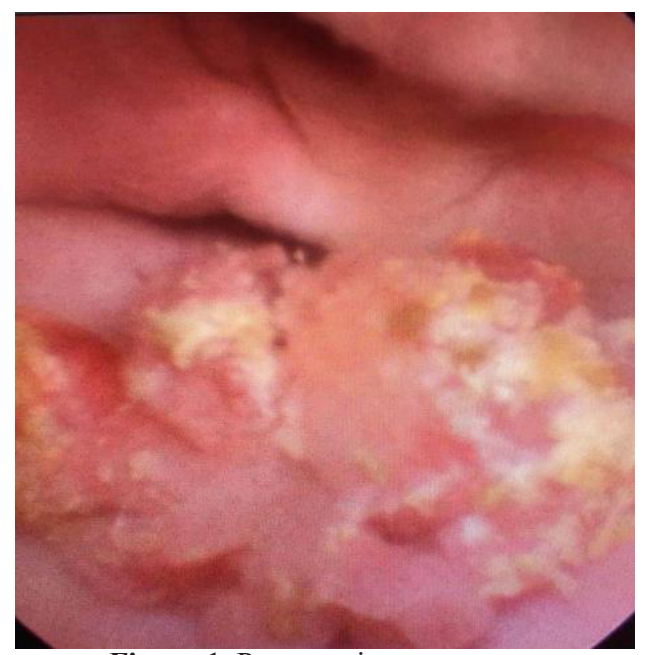

Figure 1. Preoperative cystoscopy

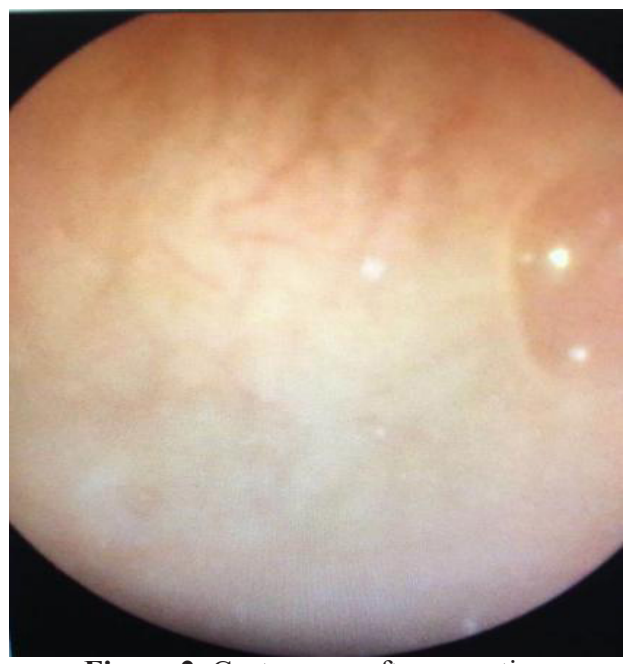

Figure 2. Cystoscopy after operation

\section{Discussion}

Among the bladder tumors, $90 \%$ were urothelial tumors, and $70 \%-80 \%$ were superficial bladder tumors ${ }^{[3]}$. Superficial bladder tumor is characterized by local infiltration, distant metastasis and recurrent recurrence, which determines that it is not easy to completely cure $^{[4]}$. At present, transurethral resection of bladder tumor (TURBT) is the main method for the treatment of bladder tumor in China. However, superficial bladder tumor is mostly multiple lesions and often complicated with carcinoma in situ. It is difficult to detect bladder cancer by naked eyes when performing cystoscopy or transurethral resection, which leads to missed diagnosis of bladder cancer and difficult to completely remove all lesions during operation. As a result, the recurrence rate of superficial bladder cancer within 2 years after surgery reaches $60 \%-70 \%$. Although intravesical instillation of chemotherapy drugs or BCG vaccine is used to reduce the recurrence rate, the effect is not ideal, and more effective treatment has been sought in clinical practice ${ }^{[5]}$.

Photodynamic therapy (PDT) is a new medical method for the treatment of tumor. PDT is a kind of photochemical therapy. The occurrence of photodynamic therapy needs photosensitizer, appropriate wavelength of light, a certain concentration of oxygen and appropriate temperature and other conditions at the same time ${ }^{[6]}$. PDT is the use of tumor cells to absorb and retain photosensitizers. Under certain light wavelength, photosensitizers produce * reactive oxygen in the light. Reactive oxygen species are cytotoxic, which can kill tumor cells and cure tumor $^{[7]}$. 
Compared with traditional tumor therapy, PDT has the advantages of lower systemic toxicity and selective destruction of tumor cells. Therefore, PDT has been used in the treatment of bladder tumors and other endoscopic accessible tumors. Since about $70 \%$ of patients with bladder cancer recur within two years after the first routine surgical treatment, if transurethral resection alone fails in chemotherapy and BCG immunotherapy, PDT can be selected to treat bladder tumor ${ }^{[8]}$. PDT is a suitable choice for superficial and multiple recurrent bladder tumors. Bladder is a cystic organ and an ideal organ for PDT. Photosensitizer can be easily injected into bladder, and targeted photosensitizer can fill the whole bladder and be absorbed by bladder tumor ${ }^{[9]}$. And light can irradiate the whole bladder, which can effectively treat the tumor cells in the bladder.PDT has its unique advantages in the treatment of bladder tumor. (1)The bladder is a cavity organ, and the photosensitizer can choose intravesical instillation to reduce the impact on other organs ${ }^{[10]}$. (2)Photosensitizers have strong selectivity for tumor cells, and they will only be absorbed and retained by tumor cells.Light exposure:It will not damage normal tissue cells ${ }^{[11]}$. (3) The treatment time is relatively short and the effect is fast ${ }^{[12]}$. (4) Intravesical instillation of photosensitizer can effectively reduce phototoxicity. (5) The incidence of postoperative adverse reactions and recurrence was reduced ${ }^{[7]}$. (6) It can retain bladder organs and ensure the body function of patients to the maximum extent ${ }^{[13]}$.

Photosensitizer is the core material of PDT. The selection of photosensitizer should meet the requirements of high selectivity to tumor cells, rapid metabolism in vivo, easy mass synthesis, good water solubility, and rapid absorption by the body. This patient was treated with phoxim, $50 \mathrm{mg}$ of which was dissolved in $50 \mathrm{ml}$ normal saline and then perfused into the bladder. As the first generation of traditional hematoporphyrin photosensitizer, hiporfin has been used in clinical treatment of malignant tumors. For the selection of photosensitizers, the most widely used photosensitizers at home and abroad are $\mathrm{HpD}$ and 5-ALA. CDHS801 is a scientific research project achievement during the Seventh Five Year Plan period in China. It belongs to the second generation photosensitizer. It is a compound extracted and separated from silkworm excrement of traditional Chinese medicine. It has the advantages of wide source of raw materials and low price ${ }^{[7]}$. Domestic scholars
Fang Jie et al $\left[^{15]}\right.$ have also achieved satisfactory results in the clinical application of CDHS801 in the treatment of bladder cancer. The design of the third generation photosensitizer is aimed at the shortcomings of the second generation photosensitizer. It is a derivative of the second generation photosensitizer, which further improves the selectivity of target tissues. At present, the third generation photosensitizer is in the animal research stage, such as crosslinked with various substances and phthalocyanines ${ }^{[16]}$.

PDT is an innovative method for the treatment of tumor. More and more clinical studies have proved the feasibility of PDT. Because of its safety, noninvasive (minimally invasive) and good selectivity, PDT will gradually become an important means of clinical treatment of bladder tumor ${ }^{[17]}$. At the same time, continuous innovation and development should be carried out to formulate the standard process and efficacy judgment standard of PDT surgery, optimize the selection of photosensitizer, reduce the adverse reactions after operation, and build a PDT medical system with better safety, more accurate target, higher cure rate and less side effects ${ }^{[18]}$.

\section{Reference:}

[1] Chen G. Photodynamic therapy and its application in bladder tumor[C].Shanghai Laser society, Shanghai Laser society, 2015.

[2] Han SJ, Zhang SJ, Chen WQ, et al. Analysis of the incidence and epidemic trend of bladder cancer in China[J]. Cancer progress, 2013,11(1): 89-95.

[3] Gu LJ. Photodynamic therapy for superficial bladder cancer [J]. Foreign medicine (Oncology Division), 2003(1): 70-73.

[4] Chen W, Bo JJ. Photodynamic therapy of bladder cancer [J]. Chinese Journal of laser medicine, 2008(2): 129-132.

[5] Zhong DL, Zeng GH. Photodynamic therapy for superficial bladder cancer [J]. Foreign medicine Urology, 2002(0): 43-46.

[6] Chen Y, Zheng J, Tan X. Research progress of photodynamic therapy in tumor therapy[J]. Journal of practical medicine, 2019(16).

[7] Zhang YF. Research and application of photodynamic therapy for bladder cancer [C]. Shanghai Laser society. Proceedings of 2005 annual meeting of Shanghai Laser society. Shanghai Laser Society: Shanghai Laser society, 2005: 13-16.

[8] Yavari N, Andersson-Engels S, Segersten U, et al. An overview on preclinical and clinical experiences with photodynamic therapy for bladder cancer[J]. Canadian Journal of Urology, 2011, 18(4): 5778-5786.

[9] Li B, Zhang YF, Chen G. Progress in photodynamic therapy for bladder cancer [J]. Journal of Clinical Urology, 2011(1): 72-74.

[10]Wang ZY, Chen G. Progress in photodynamic therapy for cavitary organ tumors [J]. Modern oncology, 2016, 24 (8): $1329-1332$. 
[11] Yang D. Progress in photodynamic therapy of tumor [J]. Applied laser, 2003(6): 378-381 + 362.

[12] Deng QZ, Zhang JG, Wang YL, et al. Transurethral resection of bladder tumor combined with photodynamic therapy to prevent postoperative recurrence[J]. Chinese Journal of physicians, 2013, 15(3): 387-389.

[13]Lee JY, Diaz RR, Cho KS, et al. Efficacy and Safety of Photodynamic Therapy for Recurrent, High Grade Nonmuscle Invasive Bladder Cancer Refractory or Intolerant to Bacille Calmette-Guérin Immunotherapy[J]. Journal of Urology, 2013, 190(4): 1192-1199.

[14]Cao ZG, song XD, Zhou SW. Photodynamic therapy and its application in bladder cancer[J]. Journal of Clinical Urology,
2006(3): 237-240.

[15]Fang J, Fang ZJ, Zhang YF. Efficacy and safety of CDHs 801 in photodynamic treatment of bladder cancer [J]. Shanghai Medical Journal, 2003(5): 306-308.

[16]Chen JB, Ou JM, Li ZQ, et al. Research progress of photodynamic drugs photosensitizers[J]. Taiwan pharmaceutical, 2003(6): 10-14.

[17]Liu JW, Yang YQ, Zhang ZG. New progress of laser photodynamic therapy for cancer [J]. Journal of Harbin Institute of technology, 2006(3): 362-366 + 373.

[18]Zhao X, Zhao KC, Liu QL, et al. Research progress of precision treatment of photodynamic tumor[J]. Acta Analytical Sciences, 2019, 35(6): 741-746. 